\title{
SOME CARTESIAN PRODUCTS OF A PATH AND PRISM RELATED GRAPHS THAT ARE EDGE ODD GRACEFUL
}

\author{
Yeni Susanti ${ }^{1 *}$, Iwan Ernanto ${ }^{1}$, Aluysius Sutjijana ${ }^{1}$, Sufyan Sidiq ${ }^{1}$ \\ ${ }^{1}$ Mathematics Department, Fac. of Mathematics and Natural Sciences, Universitas Gadjah Mada, Yogyakarta \\ Email: ${ }^{1}$ yeni_math@ugm.ac.id, ${ }^{2}$ iwan.ernanto@ugm.ac.id, ${ }^{3}$ sutjijana@ugm.ac.id, ${ }^{4}$ sufyan.sidiq@ mail.ugm.ac.id \\ *Corresponding author
}

\begin{abstract}
Let $G$ be a connected undirected simple graph of size $q$ and let $k$ be the maximum number of its order and its size. Let $f$ be a bijective edge labeling which codomain is the set of odd integers from 1 up to $2 q-1$. Then $f$ is called an edge odd graceful on $G$ if the weights of all vertices are distinct, where the weight of a vertex $v$ is defined as the sum $\bmod (2 k)$ of all labels of edges incident to $v$. Any graph that admits an edge odd graceful labeling is called an edge odd graceful graph. In this paper, some new graph classes that are edge odd graceful are presented, namely some cartesian products of path of length two and some circular related graphs.
\end{abstract}

Keywords: edge odd graceful graphs, edge odd graceful labeling, cycle, prism graphs, antiprism graphs, path, cartesian product.

\section{INTRODUCTION}

Labeling, according to Walis, [18], is a function from the set of graph elements (vertices or edges) to a set of numbers, that are usually integers. A labeling which domain is the set of edges (vertices or edges and vertices), is respectively called edge (vertex or total) labeling. It is known from the survey presented in [6] that some sorts of labeling have been defined since 1960. Many kind of labelings, including harmonious labeling, graceful labeling, anti magic labeling, magic labeling, geometric labeling, mean labeling and irregular labeling, etc. are mentioned in the survey. And for those mentioned labeling, many classes of graphs have been investigated, for instance in [1], [2], [4], [5], [7], [8], [9], [10], [11], [12], [13], [14], [15], [16]. One other labeling called edge odd graceful labeling is also introduced, by Solairaju et.all [16]. Let $G=$ $(V(G), E(G))$ be a simple undirected graph of order $n$ and of size $m$ and let $k=\max \{n, m\}$ and let $f: E(G) \longrightarrow\{1,3, \ldots, 2 m-1\}$ be an edge labeling on $G$. For any vertex $v \in V(G)$ it is defined the weight $w t_{f}(v)$ of $v$ respect to $f$ by $w t_{f}(v)=\left(\sum_{v w \in E(G)} f(v w)\right) \bmod (2 k)$. Then $f$ is called edge odd graceful labeling on $G$ if for every two vertices $v_{1}$ and $v_{2}$, the weights $w t_{f}\left(v_{1}\right)$ and $w t_{f}\left(v_{2}\right)$ are distinct. Graph $G$ is called edge odd graceful graph if $G$ admits an edge odd graceful labeling In [5], it is shown that some classes of graphs are edge odd graceful, namely, wheel graphs, double wheel graphs, web graphs, helm graphs, gear graphs, fan graphs, double fan graphs and polar grid graphs.

\section{Preliminaries}

In this section we give some preliminary terminologies and definitions. All graphs in this paper is simple and undirected. Let $G_{1}$ and $G_{2}$ be two graphs. The cartesian product of $G_{1}$ and $G_{2}$, denoted by $G_{\times} G_{2}$ is a graph with vertex set $V\left(G_{1}\right) \times V\left(G_{2}\right)$ and two arbitrary vertices 
$\left(x_{1}, y_{1}\right)$ and $\left(x_{2}, x_{2}\right)$ in $V\left(G_{1} \times G_{2}\right)$ are connected by an edge if and only if $x_{1}=x_{2}$ and $y_{1} y_{2} \in E\left(G_{2}\right)$ or $y_{1}=y_{2}$ and $x_{1} x_{2} \in E\left(G_{2}\right)$. As an example, see Figure 4 .. By path graph $P_{n}$ of order $n$, we mean a connected graph of $n$ vertices, such that two of them are of degree 1 and the remaining vertices are of degree 2. (See Figure 1.). By a cycle $C_{n}$ of order $n$ we mean a connected 2-regular graph as we can see in Figure 2..

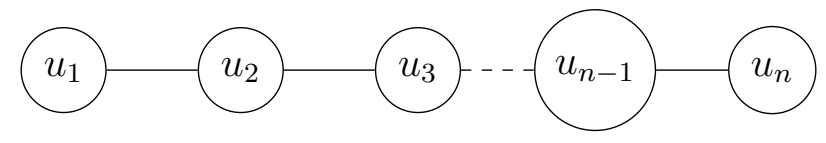

Figure 1. Path $P_{n}$

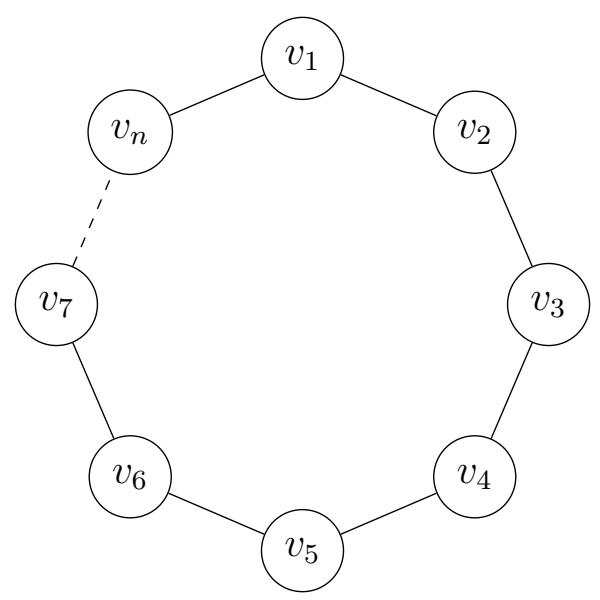

Figure 2. Cycle $C_{n}$

Let the vertex sets of $P_{2}$ and $C_{3}$ be $V\left(P_{2}\right)=\left\{u_{1}, u_{2}\right\}$ and $V\left(C_{3}\right)=\left\{v_{1}, v_{2}, v_{3}\right\}$, respectively. The cartesian product $P_{2} \times C_{3}$ is given in the Figure 3 ..

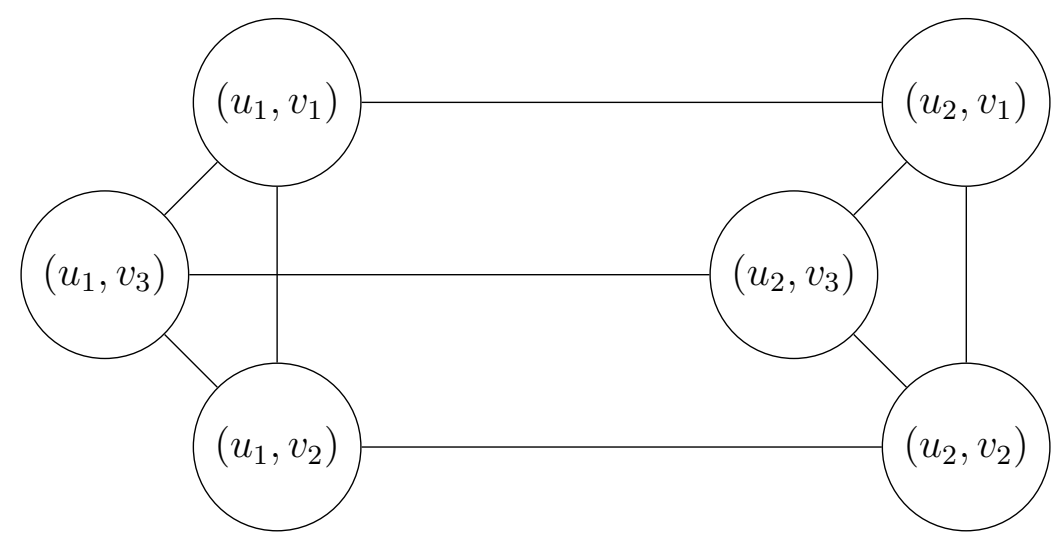

Figure 3. Cartesian Product Graph $P_{2} \times C_{3}$

In this paper, we consider some cartesian products of path of length two and some graphs, namely cycle, prism graphs, antiprism graphs and double sun flower graphs. We prove that the resulted cartesian product graphs are edge odd graceful. 


\section{RESULTS}

For the first observation, we investigate the cartesian product of path of length two with a cycle.

\subsection{Cartesian Product of Path $P_{2}$ and Cycle $C_{n}$}

Below we prove that the cartesian product of path $P_{2}$ and cycle $C_{n}, n \geq 3$ is edge odd graceful.

Theorem 1 Let $n \geq 3$ be an arbitrary positive integer. Then the graph $P_{2} \times C_{n}$ is edge odd graceful.

Proof. Let the vertex set and the edge set of $P_{2} \times C_{n}$ be

$$
V\left(P_{2} \times C_{n}\right)=\left\{a_{i}, b_{i} \mid i=1,2, \ldots, n\right\}
$$

and

$$
E\left(P_{2} \times C_{n}\right)=\left\{a_{i} a_{(i+1) \operatorname{modn}}, b_{i} b_{(i+1) \operatorname{modn}}, a_{i}, b_{i} \mid i=1,2, \ldots, n\right\},
$$

respectively. It is clear that $\left|V\left(P_{2} \times C_{n}\right)\right|=2 n$ and $\left|E\left(P_{2} \times C_{n}\right)\right|=3 n$ so that we have $k=\max \left|V\left(P_{2} \times C_{n}\right)\right|,\left|E\left(P_{2} \times C_{n}\right)\right|=3 n$. We define an edge odd labeling

$$
f_{1}: E\left(P_{2} \times C_{n}\right) \rightarrow\{1,3, \ldots, 6 n-1\}
$$

in the following manner :

$$
\begin{array}{ll}
f\left(a_{i} a_{(i+1) \operatorname{modn}}\right) & =2 i-1 \\
f\left(b_{i} b_{(i+1) \operatorname{modn}}\right) & =2 n+2 i-1 \\
f\left(a_{i} b_{i}\right) & =6 n-2 i+1
\end{array}
$$

for all $i=1,2, \ldots, n$. It is clear that

$$
\begin{array}{ll}
\left\{f\left(a_{i} a_{(i+1) \bmod n}\right) \mid i=1,2, \ldots, n\right\} & =\{1,3, \ldots, 2 n-1\} \\
\left\{f\left(b_{i} b_{(i+1) \bmod n}\right) \mid i=1,2, \ldots, n\right\} & =\{2 n+1,2 n+3, \ldots, 4 n-1\} \\
\left\{f\left(a_{i} b_{i}\right) \mid i=1,2, \ldots, n\right\} & =\{4 n+1,4 n+3, \ldots, 6 n-1\} .
\end{array}
$$

Therefore, $f_{1}$ is a bijection. Moreover, we have the following vertex weights $\bmod 6 n$ :

$$
\begin{array}{ll}
w t_{f_{1}}\left(a_{1}\right)=2 n-1 & \\
w t_{f_{1}}\left(b_{1}\right)=6 n-1 & \\
w t_{f_{1}}\left(a_{i}\right)=2 i-3, & \text { for } i=2,3, \ldots, n \\
w t_{f_{1}}\left(b_{i}\right)=4 n+2 i-3, & \text { for } i=2,3, \ldots, n,
\end{array}
$$

which are all different. Therefore $f_{1}$ is an edge odd graceful labeling. This prove that $P_{2} \times C_{n}$ is an edge odd graceful. 
On Figure 4. we give an example of the labeling for $P_{2} \times C_{3}$. The weights of the vertices are the following:

$$
\begin{array}{ll}
w t_{f_{1}}\left(a_{1}\right)=5 & w t_{f_{1}}\left(b_{1}\right)=11 \\
w t_{f_{1}}\left(a_{2}\right)=1 & w t_{f_{1}}\left(b_{2}\right)=17 \\
w t_{f_{1}}\left(a_{3}\right)=3 & w t_{f_{1}}\left(b_{3}\right)=15 .
\end{array}
$$

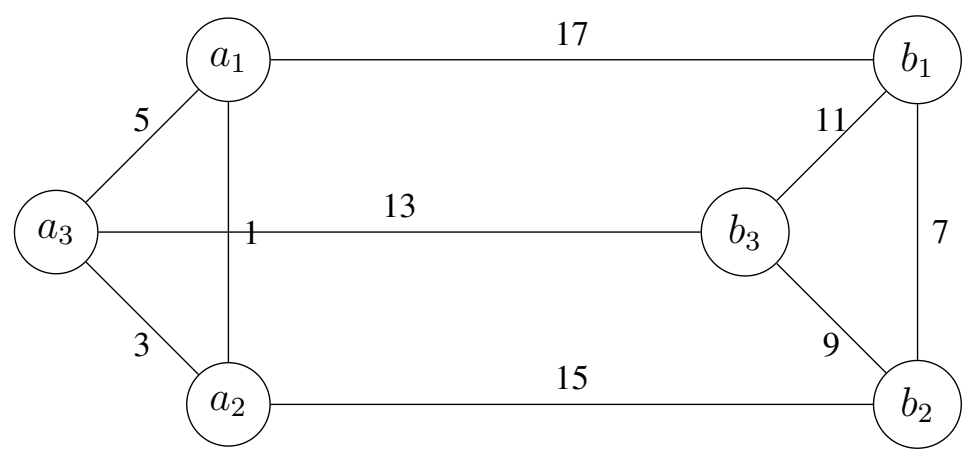

Figure 4. Cartesian Product Graph $P_{2} \times C_{3}$

\subsection{Cartesian Product of Path $P_{2}$ and Antiprism Graph $A_{n}$}

By prism graph $D_{n}$, we mean a graph of $2 n$ vertices, comprising an outer and an inner cycles of size $n$ and some additional $n$ edges connecting vertices of the outer cycle and vertices of the inner cycle (see Figure 5.). By antiprism graph $A_{n}$ we mean a graph obtained from the prism graph $D_{n}$ by adding some diagonal edges. As example of an antiprism graph, see Figure 6.

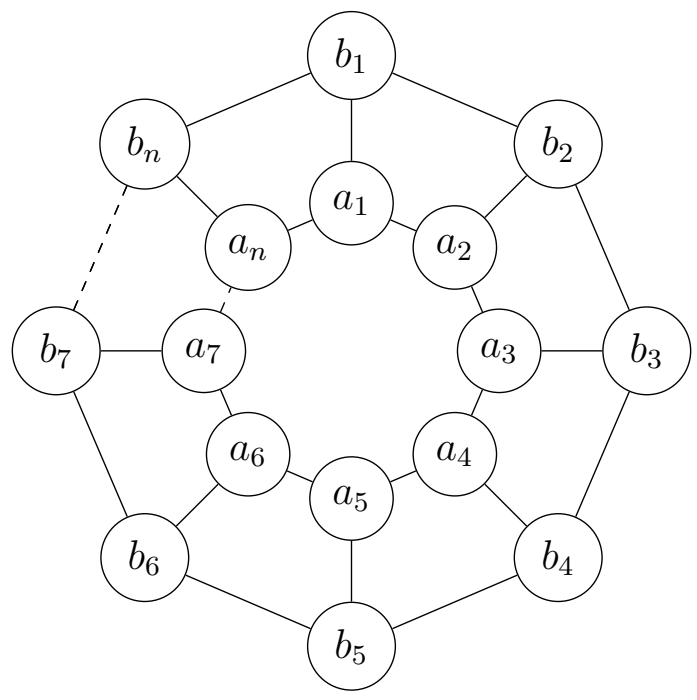

Figure 5. Prism Graph $D_{n}$

In the following theorem we prove that for arbitrary positive integer $n \geq 3$, cartesian product of path $P_{2}$ and antiprism graph $A_{n}$ is an edge odd graceful graph. 


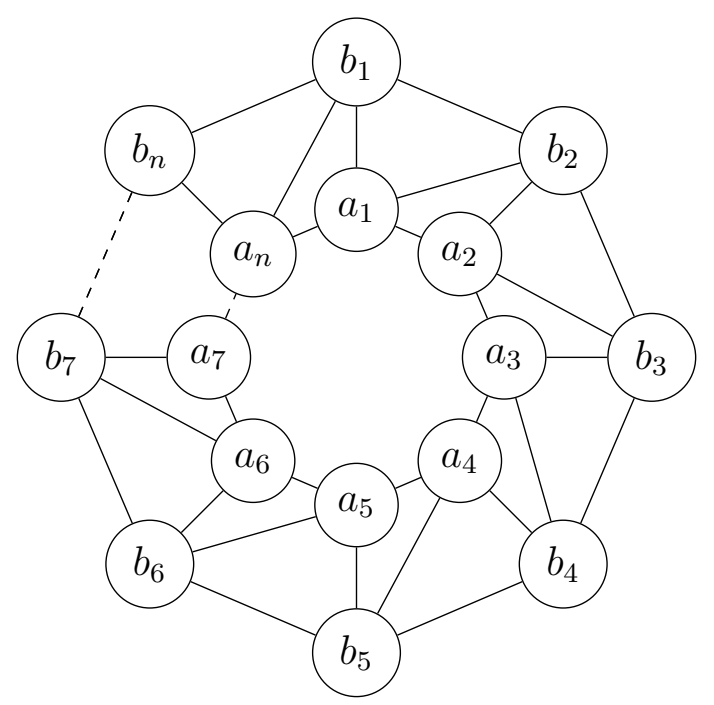

Figure 6. Antiprism Graph $A_{n}$

Theorem 2 For arbitrary positive integer $n \geq 3$, the cartesian product $P_{2} \times A_{n}$ is edge odd graceful.

Proof. Let the vertices and the edges of $P_{2} \times A_{n}$ be

$$
V\left(P_{2} \times A_{n}\right)=\left\{a_{i}, b_{i}, A_{i}, B_{i} \mid i=1,2, \ldots, n\right\}
$$

and

$$
\begin{gathered}
E\left(P_{2} \times A_{n}\right)=\left\{a_{i} a_{(i+1) \text { modn }} \mid i=1,2, \ldots, n\right\} \cup\left\{b_{i} b_{(i+1) \text { modn }} \mid i=1,2, \ldots, n\right\} \cup \\
\left\{a_{i} b_{i}, a_{i} b_{(i+1) \text { modn }} \mid i=1,2, \ldots, n\right\} \cup\left\{A_{i} A_{(i+1) \text { modn }} \mid i=1,2, \ldots, n\right\} \cup \\
\left\{B_{i} B_{(i+1) \text { modn }} \mid i=1,2, \ldots, n\right\} \cup\left\{A_{i} B_{i}, A_{i} B_{(i+1) \text { modn }} \mid i=1,2, \ldots, n\right\} \cup \\
\left\{a_{i} A_{i}, b_{i} B_{i} \mid i=1,2, \ldots, n\right\} .
\end{gathered}
$$

It is clear that $\left|V\left(P_{2} \times A_{n}\right)\right|=4 n$ and $\left|E\left(P_{2} \times A_{n}\right)\right|=10 n$ and hence we have $k=$ $\max \left\{\left|V\left(P_{2} \times A_{n}\right)\right|,\left|E\left(P_{2} \times A_{n}\right)\right|\right\}=10 n$. Now we construct an edge odd labeling

$$
f_{2}: E\left(P_{2} \times A_{n}\right) \rightarrow\{1,2, \ldots, 20 n-1\}
$$

by 


$$
\begin{array}{ll}
f_{2}\left(a_{i} a_{(i+1) \operatorname{modn}}\right) & =2 n-2 i+1 \\
f_{2}\left(b_{i} b_{(i+1) \operatorname{modn}}\right) & =8 n-2 i+1 \\
f_{2}\left(a_{i} b_{(i+1) \operatorname{modn}}\right) & =14 n+2 i-1 \\
f_{2}\left(a_{i} b_{i}\right) & =8 n+2 i-1 \\
f_{2}\left(A_{i} A_{(i+1) \operatorname{modn}}\right) & =4 n+2 i-1 \\
f_{2}\left(B_{i} B_{(i+1) \operatorname{modn}}\right) & =12 n-2 i+1 \\
f_{2}\left(A_{i} B_{(i+1) \operatorname{modn}}\right) & =18 n+2 i-1 \\
f_{2}\left(A_{i} B_{i}\right) & =14 n-2 i+1 \\
f_{2}\left(a_{i} A_{i}\right) & =4 n-2 i+1 \\
f_{2}\left(b_{i} B_{i}\right) & =16 n+2 i-1
\end{array}
$$

for all $i=1,2, \ldots, n$. It is clear that

$$
\begin{array}{ll}
\left\{f_{2}\left(a_{i} a_{(i+1) \bmod n}\right) \mid i=1,2, \ldots, n\right\} & =\{1,3, \ldots, 2 n-1\} \\
\left\{f_{2}\left(b_{i} b_{(i+1) \bmod n}\right) \mid i=1,2, \ldots, n\right\} & =\{6 n+1,6 n+3, \ldots, 8 n-1\} \\
\left\{f_{2}\left(a_{i} b_{(i+1) \operatorname{modn}}\right) \mid i=1,2, \ldots, n\right\} & =\{14 n+1,14 n+3, \ldots, 16 n-1\} \\
\left\{f_{2}\left(a_{i} b_{i}\right) \mid i=1,2, \ldots, n\right\} & =\{8 n+1,8 n+3, \ldots, 10 n-1\} \\
\left\{f_{2}\left(A_{i} A_{(i+1) \bmod n}\right) \mid i=1,2, \ldots, n\right\} & =\{4 n+1,4 n+3, \ldots, 6 n-1\} \\
\left\{f_{2}\left(B_{i} B_{(i+1) \bmod n}\right) \mid i=1,2, \ldots, n\right\} & =\{10 n+1,10 n+3, \ldots, 12 n-1\} \\
\left\{f_{2}\left(A_{i} B_{(i+1) \bmod n}\right) \mid i=1,2, \ldots, n\right\} & =\{18 n+1,18 n+3, \ldots, 20 n-1\} \\
\left\{f_{2}\left(A_{i} B_{i}\right) \mid i=1,2, \ldots, n\right\} & =\{12 n+1,12 n+3, \ldots, 14 n-1\} \\
\left\{f_{2}\left(a_{i} A_{i}\right) \mid i=1,2, \ldots, n\right\} & =\{2 n+1,2 n+3, \ldots, 4 n-1\} \\
\left\{f_{2}\left(b_{i} B_{i}\right) \mid i=1,2, \ldots, n\right\} & =\{16 n+1,16 n+3, \ldots, 18 n-1\}
\end{array}
$$

confirming that $f_{2}$ is a bijection. Furthermore we then obtain the following vertex weights $\bmod 2 k$ for $2 k=20 n$ under the labeling $f_{2}$ :

$$
\begin{array}{lr}
w t_{f_{3}}\left(a_{1}\right)=8 n+1 & \\
w t_{f_{3}}\left(a_{i}\right)=10 n-2 i+3 & \text { for } i=2,3, \ldots, n \\
w t_{f_{3}}\left(b_{1}\right)=14 n+1 & \\
w t_{f_{3}}\left(b_{i}\right)=14 n+2 i-1 & \text { for } i=2,2, \ldots, n \\
w t_{f_{3}}\left(A_{1}\right)=6 n-1 & \\
w t_{f_{3}}\left(A_{i}\right)=4 n+2 i-3 & \text { for } i=2,3, \ldots, n \\
w t_{f_{3}}\left(B_{1}\right)=12 n-1 & \\
w t_{f_{3}}\left(B_{i}\right)=12 n-2 i+1 & \text { for } i=2,3, \ldots, n .
\end{array}
$$

It is easy to check that 


$$
\begin{array}{ll}
\left\{w t_{f_{3}}\left(a_{1}\right)\right\} & =\{8 n+1\} \\
\left\{w t_{f_{3}}\left(a_{i}\right) \mid i=2,3, \ldots, n\right\} & =\{8 n+3,8 n+5, \ldots, 10 n-1\} \\
\left\{w t_{f_{3}}\left(b_{1}\right)\right\} & =\{14 n+1\} \\
\left\{w t_{f_{3}}\left(b_{i}\right) \mid i=2,3, \ldots, n\right\} & =\{14 n+3,14 n+5, \ldots 16 n-1\} \\
\left\{w t_{f_{3}}\left(A_{1}\right)\right\} & =6 n-1 \\
\left\{w t_{f_{3}}\left(A_{i}\right) \mid i=2,3, \ldots, n\right\} & =\{4 n+1,4 n+3, \ldots, 6 n-3\} \\
\left\{w t_{f_{3}}\left(B_{1}\right)\right\} & =12 n-1 \\
\left\{w t_{f_{3}}\left(B_{i}\right) \mid i=2,3, \ldots, n\right\} & =\{10 n+1,10 n+3, \ldots, 12 n-3\}
\end{array}
$$

showing that the weights are all distinct. Hence $f_{2}$ is an edge odd graceful on $P_{2} \times A_{n}$ and therefore $P_{2} \times A_{n}$ is an edge oddd graceful.

In Figure 7., we give an illustration of an edge odd labeling on $P_{2} \times A_{8}$. We separate the graph into several part to make the labeling look clear.

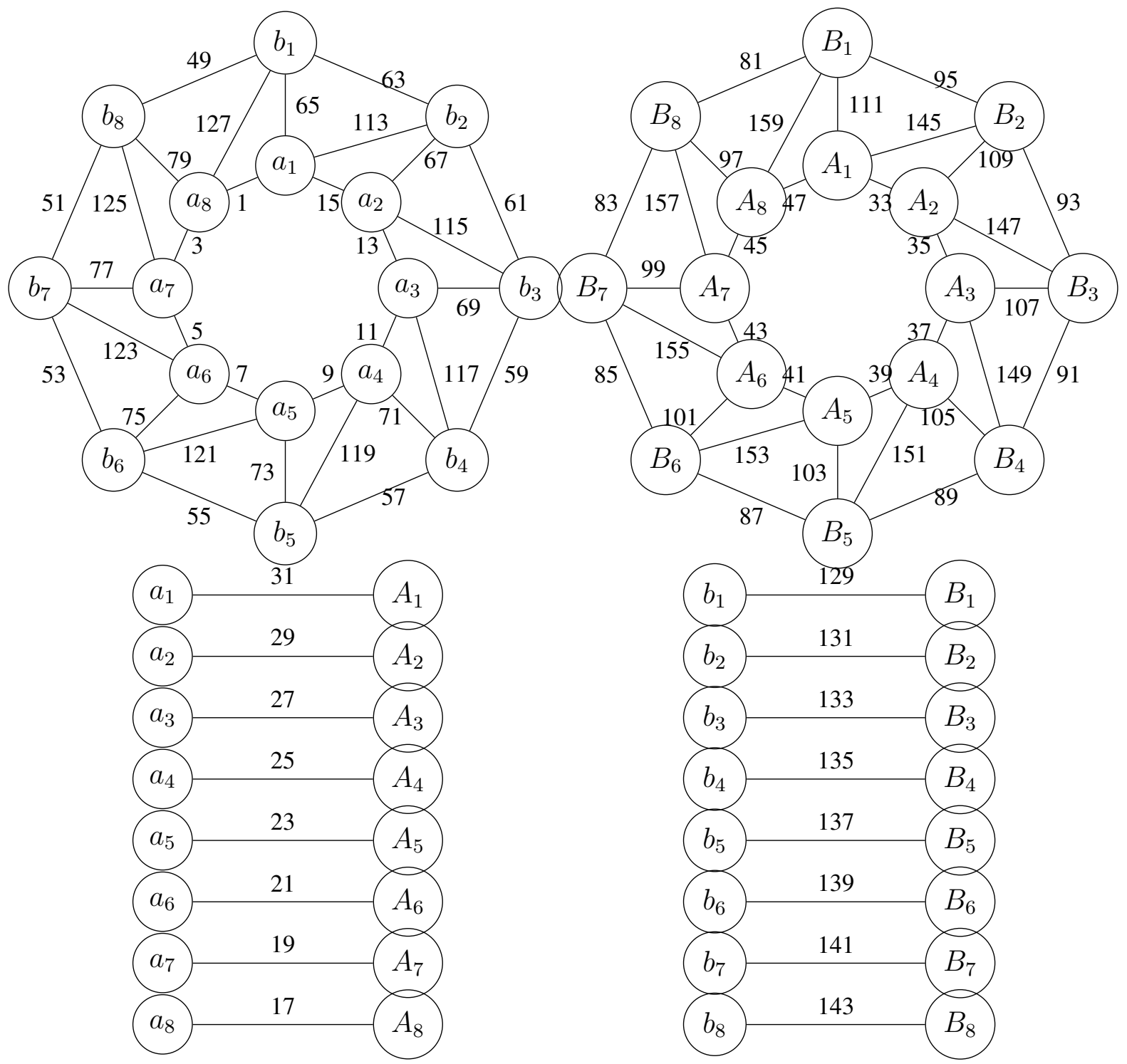

Figure 7. Edge Odd Graceful Labeling on Cartesian Product $P_{2} \times A_{8}$ 
From the Figure 7., we obtain vertex weights $\bmod 160$ respect to labeling $f_{2}$ as follows:

$$
\begin{array}{llll}
w t_{f_{3}}\left(a_{1}\right)=65 & w t_{f_{3}}\left(b_{1}\right)=113 & w t_{f_{3}}\left(A_{1}\right)=47 & w t_{f_{3}}\left(B_{1}\right)=95 \\
w t_{f_{3}}\left(a_{2}\right)=79 & w t_{f_{3}}\left(b_{2}\right)=115 & w t_{f_{3}}\left(A_{2}\right)=33 & w t_{f_{3}}\left(B_{2}\right)=93 \\
w t_{f_{3}}\left(a_{3}\right)=77 & w t_{f_{3}}\left(b_{3}\right)=117 & w t_{f_{3}}\left(A_{3}\right)=35 & w t_{f_{3}}\left(B_{3}\right)=91 \\
w t_{f_{3}}\left(a_{4}\right)=75 & w t_{f_{3}}\left(b_{4}\right)=119 & w t_{f_{3}}\left(A_{4}\right)=37 & w t_{f_{3}}\left(B_{4}\right)=89 \\
w t_{f_{3}}\left(a_{5}\right)=73 & w t_{f_{3}}\left(b_{5}\right)=121 & w t_{f_{3}}\left(A_{5}\right)=39 & w t_{f_{3}}\left(B_{5}\right)=87 \\
w t_{f_{3}}\left(a_{6}\right)=71 & w t_{f_{3}}\left(b_{6}\right)=123 & w t_{f_{3}}\left(A_{6}\right)=41 & w t_{f_{3}}\left(B_{6}\right)=85 \\
w t_{f_{3}}\left(a_{7}\right)=69 & w t_{f_{3}}\left(b_{7}\right)=125 & w t_{f_{3}}\left(A_{7}\right)=43 & w t_{f_{3}}\left(B_{7}\right)=83 \\
w t_{f_{3}}\left(a_{8}\right)=67 & w t_{f_{3}}\left(b_{8}\right)=127 & w t_{f_{3}}\left(A_{8}\right)=45 & w t_{f_{3}}\left(B_{8}\right)=81 .
\end{array}
$$

\subsection{Cartesian Product of Path $P_{2}$ and Double Sun Flower $D S F_{n}$}

Definition 1 A sun flower graph of order $2 n$, denoted by $S F_{n}$, is a graph that is isomorphic to a graph obtained by deleting edges of the outer cycle from antiprism graph $A_{n}$ (see FIGURE 8.).

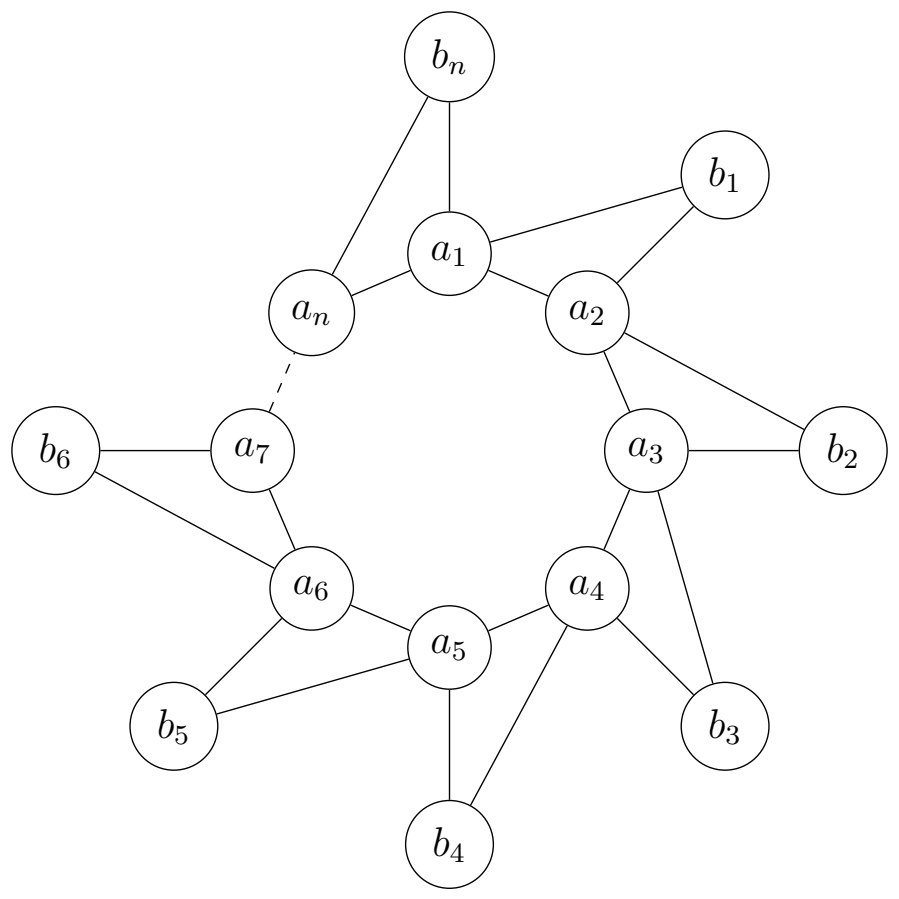

Figure 8. Sun Flower Graph $S F_{n}$

The vertex set and the edge set of $S F_{n}$ of order $2 n$, are, respectively

$$
V\left(S F_{n}\right)=\left\{a_{i}, b_{i} \mid i=1,2, \ldots, n\right\}
$$

and

$$
E\left(S F_{n}\right)=\left\{a_{i} a_{(i+1) \bmod n}, a_{i} b_{i}, a_{(i+1) \bmod n} b_{i} \mid i=1,2, \ldots, n\right\} .
$$

Thus we have $\left|V\left(S F_{n}\right)\right|=2 n$ and $\left|E\left(S F_{n}\right)\right|=3 n$. 
Definition 2 By a double sun flower graph of order $3 n$, denoted by $D S F_{n}$, is a graph obtained from the graph $S F_{n}$ (see FIGURE 8.) by inserting a new vertex $c_{i}$ on each edges $a_{i} a_{i+1}$ and adding edges $b_{i} c_{i}$ for each $i$. (See FIGURE 9.)

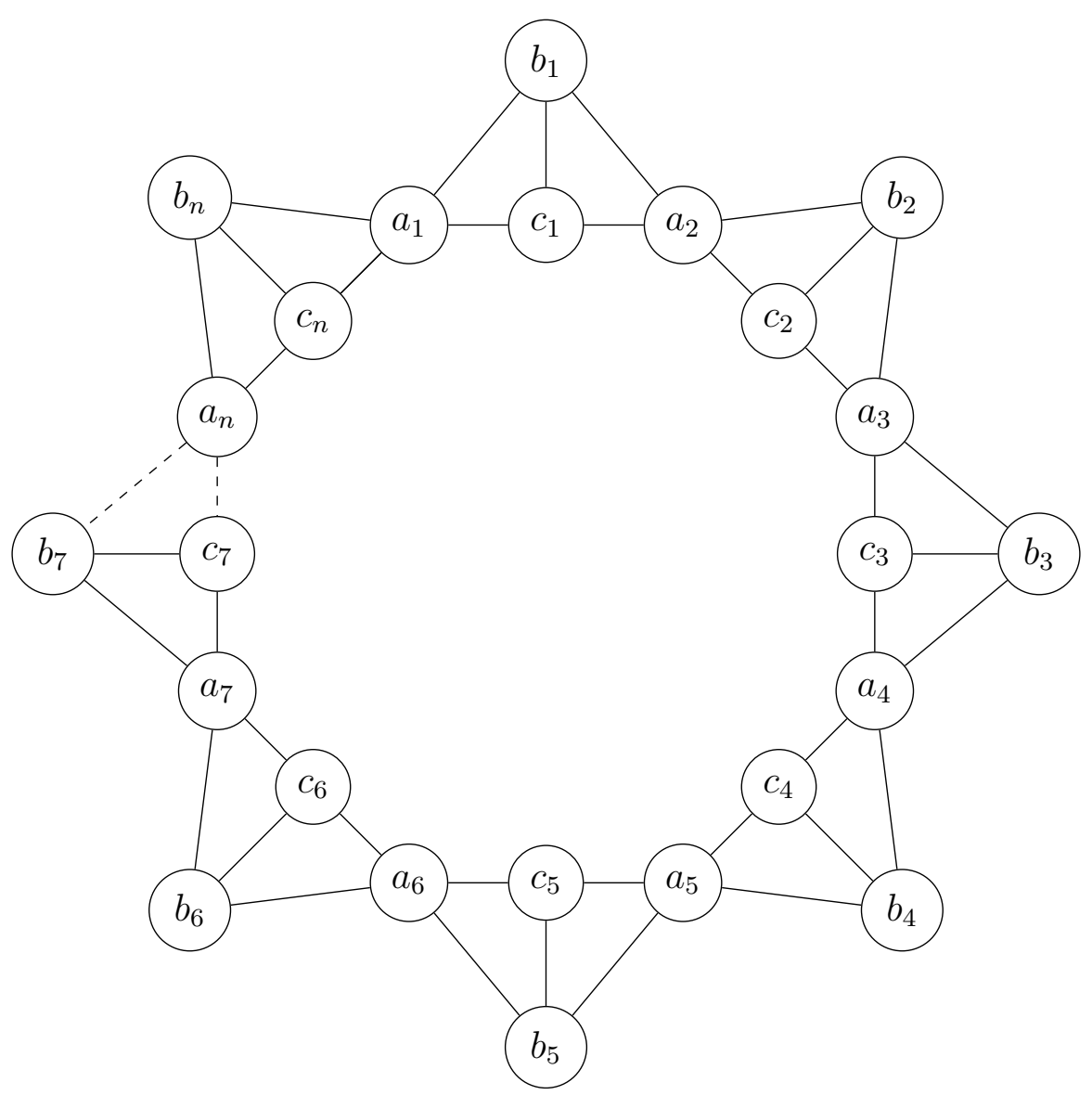

Figure 9. Double Sun Flower Graph $D S F_{n}$

Theorem 3 Let $n \geq 3$ be an arbitrary positive integer. Then the graph $P_{2} \times D S F_{n}$ is edge odd graceful.

Proof. Let the vertex set and the edge set of $P_{2} \times D S F_{n}$ be

$$
V\left(P_{2} \times D S F_{n}\right)=\left\{a_{i}, b_{i}, c_{i}, A_{i}, B_{i}, C_{i} \mid i=1,2, \ldots, n\right\}
$$

and

$$
\begin{gathered}
E\left(P_{2} \times D S F_{n}\right)=\left\{a_{i} b_{i}, a_{i} c_{i}, b_{i} c_{i}, b_{i} a_{(i+1) \operatorname{modn}}, c_{i} a_{(i+1) \operatorname{modn}} \mid i=1,2, \ldots, n\right\} \cup \\
\left\{A_{i} B_{i}, A_{i} C_{i}, B_{i} C_{i} \mid i=1,2, \ldots, n\right\} \cup \\
\left\{B_{i} A_{(i+1) \operatorname{modn}}, C_{i} A_{(i+1) \operatorname{modn}}, a_{i} A_{i}, b_{i} B_{i}, c_{i} C_{i} \mid i=1,2, \ldots, n\right\} .
\end{gathered}
$$

It is clear that

$$
\left|V\left(P_{2} \times D S F_{n}\right)\right|=6 n
$$


and

$$
\left|E\left(P_{2} \times D S F_{n}\right)\right|=13 n .
$$

We construct an edge labeling $f_{3}: E\left(P_{2} \times D S F_{n}\right) \rightarrow\{1,3, \ldots, 26 n-1\}$ for two cases, whenever $n$ is odd and $n$ is even.

\section{Case for $n$ odd}

We define the labeling as follows:

$$
\begin{array}{ll}
f_{3}\left(a_{i} b_{i}\right) & =20 n-2 i+1 \\
f_{3}\left(a_{i} c_{i}\right) & =16 n-2 i+1 \\
f_{3}\left(b_{i} c_{i}\right) & =12 n+2 i-1 \\
f_{3}\left(b_{i} a_{(i+1) \operatorname{modn}}\right) & =10 n+2 i-1 \\
f_{3}\left(c_{i} a_{(i+1) \text { modn }}\right) & =18 n-2 i+1 \\
f_{3}\left(A_{i} B_{i}\right) & =10 n-2 i+1 \\
f_{3}\left(A_{i} C_{i}\right) & =6 n-2 i+1 \\
f_{3}\left(B_{i} C_{i}\right) & =22 n+2 i-1 \\
f_{3}\left(B_{i} A_{(i+1) \operatorname{modn}}\right) & =2 i-1 \\
f_{3}\left(C_{i} A_{(i+1) \operatorname{modn}}\right) & =8 n-2 i+1 \\
f_{3}\left(a_{i} A_{i}\right) & =20 n+2 i-1 \\
f_{3}\left(b_{i} B_{i}\right) & =2 n+2 i-1 \\
f_{3}\left(c_{i} C_{i}\right) & =26 n-2 i+1
\end{array}
$$

for all $i=1,2, \ldots, n$. It is easy to see that

$$
\begin{array}{ll}
\left\{f_{3}\left(a_{i} b_{i}\right) \mid i=1,2, \ldots, n\right\} & =\{18 n+1,18 n+3, \ldots, 20 n-1\} \\
\left\{f_{3}\left(a_{i} c_{i}\right) \mid i=1,2, \ldots, n\right\} & =\{14 n+1,14 n+3, \ldots, 16 n-1\} \\
\left\{f_{3}\left(b_{i} c_{i}\right) \mid i=1,2, \ldots, n\right\} & =\{12 n+1,12 n+3, \ldots, 14 n-1\} \\
\left\{f_{3}\left(b_{i} a_{(i+1) \text { modn }}\right) \mid i=1,2, \ldots, n\right\} & =\{10 n+1,10 n+3, \ldots, 12 n-1\} \\
\left\{f_{3}\left(c_{i} a_{(i+1) \text { modn }}\right) \mid i=1,2, \ldots, n\right\} & =\{16 n+1,16 n+3, \ldots, 18 n-1\} \\
\left\{f_{3}\left(A_{i} B_{i}\right) \mid i=1,2, \ldots, n\right\} & =\{8 n+1,8 n+3, \ldots, 10 n-1\} \\
\left\{f_{3}\left(A_{i} C_{i}\right) \mid i=1,2, \ldots, n\right\} & =\{4 n+1,4 n+3, \ldots, 6 n-1\} \\
\left\{f_{3}\left(B_{i} C_{i}\right) \mid i=1,2, \ldots, n\right\} & =\{22 n+1,22 n+3, \ldots, 24 n-1\} \\
\left\{f_{3}\left(B_{i} A_{(i+1) \operatorname{modn}}\right) \mid i=1,2, \ldots, n\right\} & =\{1,3, \ldots, 2 n-1\} \\
\left\{f_{3}\left(C_{i} A_{(i+1) \operatorname{modn}}\right) \mid i=1,2, \ldots, n\right\} & =\{6 n+1,6 n+3, \ldots, 8 n-1\} \\
\left\{f_{3}\left(a_{i} A_{i}\right) \mid i=1,2, \ldots, n\right\} & =\{20 n+1,20 n+3, \ldots, 22 n-1\} \\
\left\{f_{3}\left(b_{i} B_{i}\right) \mid i=1,2, \ldots, n\right\} & =\{2 n+1,2 n+3, \ldots, 4 n-1\} \\
\left\{f_{3}\left(c_{i} C_{i}\right) \mid i=1,2, \ldots, n\right\} & =\{24 n+1,24 n+3, \ldots, 26 n-1\} .
\end{array}
$$

Thus $f_{3}$ is a bijection. Moreover, we then have vertex weights $\bmod 2 k$, where $k=\max \left\{\mid V\left(P_{2} \times\right.\right.$ $\left.\left.D S F_{n}\right)|| E,\left(P_{2} \times D S F_{n}\right) \mid\right\}=\max \{5 n, 13 n\}=13 n$ as follows: 


$$
\begin{array}{lll}
w t_{f_{3}}\left(a_{1}\right)=6 n-1 & w t_{f_{3}}\left(A_{1}\right)=18 n-1 & \\
w t_{f_{3}}\left(a_{i}\right)=6 n-2 i+1 & w t_{f_{3}}\left(A_{i}\right)=18 n-2 i+1 & \text { for } i=2,3, \ldots, n \\
w t_{f_{3}}\left(b_{i}\right)=18 n+4 i-2 & w t_{f_{3}}\left(B_{i}\right)=8 n+4 i-2 & \text { for } i=1,2, \ldots, n \\
w t_{f_{3}}\left(c_{i}\right)=20 n-4 i+2 & w t_{f_{3}}\left(C_{i}\right)=10 n-4 i+2 & \text { for } i=1,2, \ldots, n
\end{array}
$$

so that

$$
\begin{array}{ll}
\left\{w t_{f_{3}}\left(a_{1}\right)\right\} & =\{6 n-1\} \\
\left\{w t_{f_{3}}\left(a_{i}\right) \mid i=2,3, \ldots, n\right\} & =\{4 n+1,4 n+3, \ldots, 6 n-3\} \\
\left\{w t_{f_{3}}\left(b_{i}\right) \mid i=1,2, \ldots, n\right\} & =\{18 n+2,18 n+6, \ldots, 22 n-1\} \\
\left\{w t_{f_{3}}\left(c_{i}\right) \mid i=1,2, \ldots, n\right\} & =\{16 n+2,16 n+6, \ldots, 20 n-2\} \\
\left\{w t_{f_{3}}\left(A_{1}\right)\right\} & =\{18 n-1\} \\
\left\{w t_{f_{3}}\left(A_{i}\right) \mid i=2,3, \ldots, n\right\} & =\{16 n+1,16 n+3, \ldots, 18 n-3\} \\
\left\{w t_{f_{3}}\left(B_{i}\right) \mid i=1,2, \ldots, n\right\} & =\{8 n+2,8 n+6, \ldots, 12 n-2\} \\
\left\{w t_{f_{3}}\left(C_{i}\right) \mid i=1,2, \ldots, n\right\} & =\{6 n+2, n+6, \ldots, 10 n-2\} .
\end{array}
$$

It is easy to check that for arbitrary odd number $n \geq 3$, all the weights are different. Therefore $f_{3}$ is an edge odd graceful labeling. Thus, for $n \geq 3$ odd, $P_{2} \times D S F_{n}$ is edge odd graceful.

\section{Case for $n$ even}

For $n \geq 3$ even we define the labeling as follows:

$$
\begin{array}{ll}
f_{3}\left(a_{i} b_{i}\right) & =16 n-2 i+1 \\
f_{3}\left(a_{i} c_{i}\right) & =20 n-2 i+1 \\
f_{3}\left(b_{i} c_{i}\right) & =2 i-1 \\
f_{3}\left(b_{i} a_{(i+1) \operatorname{modn}}\right) & =10 n+2 i-1 \\
f_{3}\left(c_{i} a_{(i+1) \operatorname{modn}}\right) & =16 n-2 i+1 \\
f_{3}\left(A_{i} B_{i}\right) & =8 n-2 i+1 \\
f_{3}\left(A_{i} C_{i}\right) & =10 n-2 i+1 \\
f_{3}\left(B_{i} C_{i}\right) & =12 n+2 i-1 \\
f_{3}\left(B_{i} A_{(i+1) \operatorname{modn}}\right) & =22 n+2 i-1 \\
f_{3}\left(C_{i} A_{(i+1) \operatorname{modn}}\right) & =18 n-2 i+1 \\
f_{3}\left(a_{i} A_{i}\right) & =20 n+2 i-1 \\
f_{3}\left(b_{i} B_{i}\right) & =2 n+2 i-1 \\
f_{3}\left(c_{i} C_{i}\right) & =26 n-2 i+1
\end{array}
$$

for all $i=1,2, \ldots, n$. We obtain 


$$
\begin{array}{ll}
\left\{f_{3}\left(a_{i} b_{i}\right) \mid i=1,2, \ldots, n\right\} & =\{14 n-1,14 n+1, \ldots, 16 n-1\} \\
\left\{f_{3}\left(a_{i} c_{i}\right) \mid i=1,2, \ldots, n\right\} & =\{18 n+1,18 n+3, \ldots, 20 n-1\} \\
\left\{f_{3}\left(b_{i} c_{i}\right) \mid i=1,2, \ldots, n\right\} & =\{1,3, \ldots, 2 n-1\} \\
\left\{f_{3}\left(b_{i} a_{(i+1) \operatorname{modn}}\right) \mid i=1,2, \ldots, n\right\} & =\{10 n+1,10 n+3, \ldots, 12 n-1\} \\
\left\{f_{3}\left(c_{i} a_{(i+1) \bmod n}\right) \mid i=1,2, \ldots, n\right\} & =\{14 n+1,14 n+3, \ldots, 16 n-1\} \\
\left\{f_{3}\left(A_{i} B_{i}\right) \mid i=1,2, \ldots, n\right\} & =\{6 n+1,6 n+3, \ldots, 8 n-1\} \\
\left\{f_{3}\left(A_{i} C_{i}\right) \mid i=1,2, \ldots, n\right\} & =\{8 n+1,8 n+3, \ldots, 10 n-1\} \\
\left\{f_{3}\left(B_{i} C_{i}\right) \mid i=1,2, \ldots, n\right\} & =\{12 n+1,12 n+3, \ldots, 14 n-1\} \\
\left\{f_{3}\left(B_{i} A_{(i+1) \operatorname{modn}}\right) \mid i=1,2, \ldots, n\right\} & =\{22 n+1,22 n+3, \ldots, 24 n-1\} \\
\left\{f_{3}\left(C_{i} A_{(i+1) \operatorname{modn}}\right) \mid i=1,2, \ldots, n\right\} & =\{16 n+1,16 n+3, \ldots, 18 n-1\} \\
\left\{f_{3}\left(a_{i} A_{i}\right) \mid i=1,2, \ldots, n\right\} & =\{20 n+1,20 n+3, \ldots, 22 n-1\} \\
\left\{f_{3}\left(b_{i} B_{i}\right) \mid i=1,2, \ldots, n\right\} & =\{2 n+1,2 n+3, \ldots, 4 n-1\} \\
\left\{f_{3}\left(c_{i} C_{i}\right) \mid i=1,2, \ldots, n\right\} & =\{24 n+1,24 n+3, \ldots, 26 n-1\} .
\end{array}
$$

We then have vertex weights $\bmod 2 k$, where $k=\max \left\{\left|V\left(P_{2} \times D S F_{n}\right)\right|,\left|E\left(P_{2} \times D S F_{n}\right)\right|\right\}=$ $\max \{5 n, 13 n\}=13 n$ as follows:

$$
\begin{array}{ll}
w t_{f_{3}}\left(a_{1}\right)=20 n-1 & \\
w t_{f_{3}}\left(a_{i}\right)=20 n-2 i+1 & \text { for } i=2,3, \ldots, n \\
w t_{f_{3}}\left(b_{i}\right)=2 n+4 i-2 & \text { for } i=1,2, \ldots, n \\
w t_{f_{3}}\left(c_{i}\right)=26 n-4 i+2 & \text { for } i=1,2, \ldots, n . \\
w t_{f_{3}}\left(A_{1}\right)=26 n-1 & \\
w t_{f_{3}}\left(A_{i}\right)=26 n-2 i+1 & \text { for } i=2,3, \ldots, n \\
w t_{f_{3}}\left(B_{i}\right)=18 n+4 i-2 & \text { for } i=1,2, \ldots, n \\
w t_{f_{3}}\left(C_{i}\right)=14 n-4 i+2 & \text { for } i=1,2, \ldots, n .
\end{array}
$$

Clearly,

$$
\begin{array}{ll}
\left\{w t_{f_{3}}\left(a_{1}\right)\right\} & =\{20 n-1\} \\
\left\{w t_{f_{3}}\left(a_{i}\right) \mid i=2,3, \ldots, n\right\} & =\{18 n+1,18 n+3, \ldots, 20 n-3\} \\
\left\{w t_{f_{3}}\left(b_{i}\right) \mid i=1,2, \ldots, n\right\} & =\{2 n+2,2 n+6, \ldots, 6 n-2\} \\
\left.\left\{w t_{f_{3}}\left(c_{i}\right) \mid i=1,2, \ldots, n\right\}\right\} & =\{22 n+2,22 n+6, \ldots, 26 n-2\} . \\
\left\{w t_{f_{3}}\left(A_{1}\right)\right\} & =\{26 n-1\} \\
\left\{w t_{f_{3}}\left(A_{i}\right) \mid i=2,3, \ldots, n\right\} & =\{24 n+1,24 n+3, \ldots, 26 n-3\} \\
\left\{w t_{f_{3}}\left(B_{i}\right) \mid i=1,2, \ldots, n\right\} & =\{18 n+2,18 n+6, \ldots, 22 n-2\} \\
\left\{w t_{f_{3}}\left(C_{i}\right) \mid i=1,2, \ldots, n\right\} & =\{10 n+2,10 n+6, \ldots, 14 n-2\} .
\end{array}
$$

Therefore $f_{3}$ is an edge odd labeling for every even number $n \geq 3$ and hence $P_{2} \times D S F_{n}$ is edge odd graceful for each even positive integer $n \geq 3$. Thus $P_{2} \times D S F_{n}$ is edge odd graceful for arbitrary positive integer $n \geq 3$.

Figure 10. shows an edge odd labeling on $P_{2} \times D S F_{3}$. To make the figure look clear, we 
draw the graph into several separate parts. Respect to the labeling $f_{3}$ for $P_{2} \times D S F_{3}$ we have

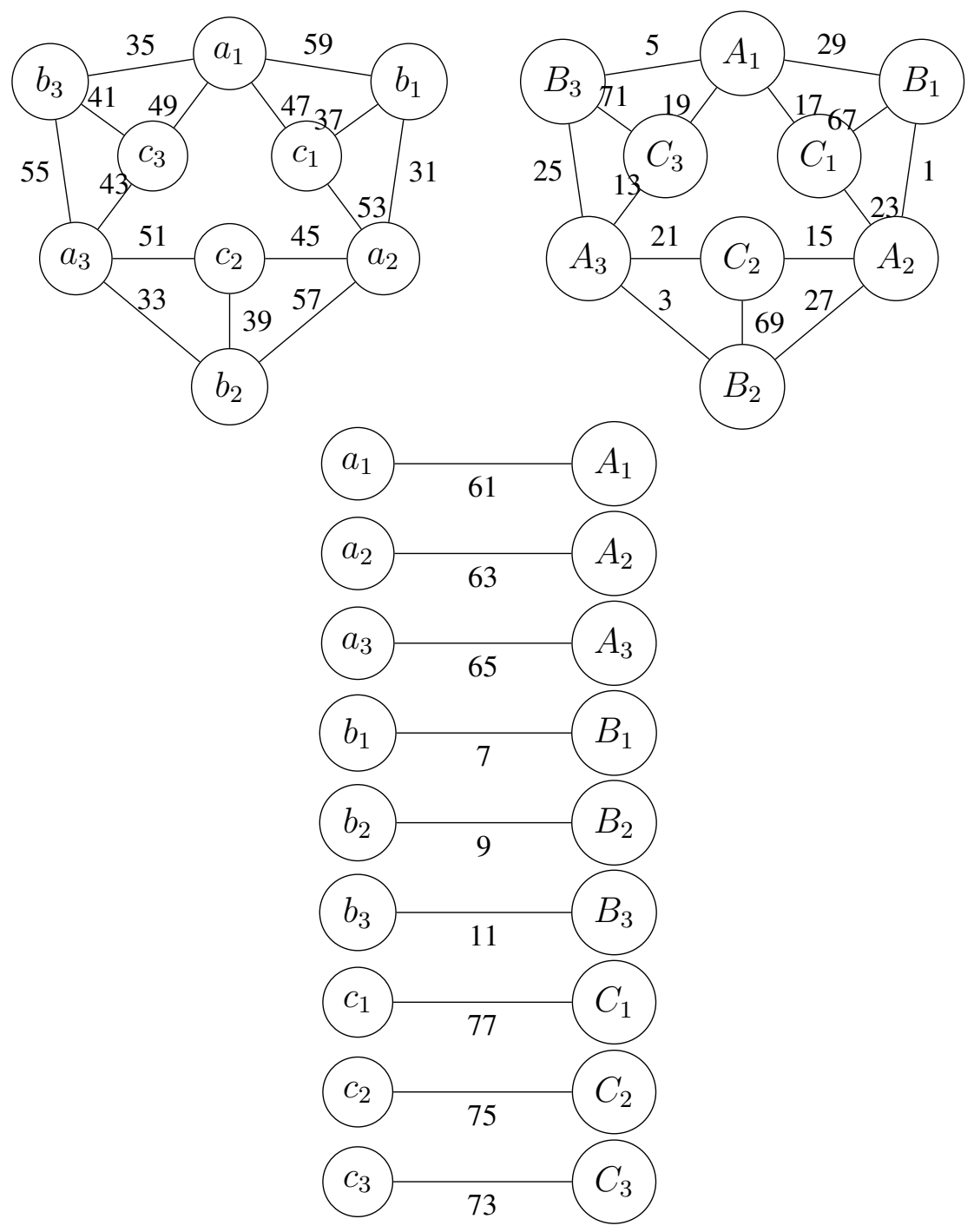

Figure 10. Edge Odd Graceful Labeling $f_{3}$ for $P_{2} \times D S F_{3}$

the following vertex weights:

$$
\begin{array}{ll}
w t_{f_{2}}\left(a_{1}\right)=17 & w t_{f_{2}}\left(A_{1}\right)=53 \\
w t_{f_{2}}\left(a_{2}\right)=15 & w t_{f_{2}}\left(A_{2}\right)=51 \\
w t_{f_{2}}\left(a_{3}\right)=13 & w t_{f_{2}}\left(A_{3}\right)=49 \\
w t_{f_{2}}\left(b_{1}\right)=56 & w t_{f_{2}}\left(B_{1}\right)=26 \\
w t_{f_{2}}\left(b_{2}\right)=60 & w t_{f_{2}}\left(B_{2}\right)=30 \\
w t_{f_{2}}\left(b_{3}\right)=64 & w t_{f_{2}}\left(B_{3}\right)=34 \\
w t_{f_{2}}\left(c_{1}\right)=58 & w t_{f_{2}}\left(C_{1}\right)=28 \\
w t_{f_{2}}\left(c_{2}\right)=54 & w t_{f_{2}}\left(C_{2}\right)=24 \\
w t_{f_{2}}\left(c_{3}\right)=50 & w t_{f_{2}}\left(C_{3}\right)=20 .
\end{array}
$$


JOURNAL OF FUNDAMENTAL MATHEMATICS

AND APPLICATIONS (JFMA) VOL. 4 NO. 2 (NOV 2021)

Available online at www.jfma.math.fsm.undip.ac.id

\section{ACKNOWLEDGEMENT}

This work was done by the support from Universitas Gadjah Mada under Research Grant Year 2019 ( Hibah Penelitian Dosen Dana Masyarakat Alokasi Fakultas Tahun 2019).

\section{REFERENCES}

[1] M. Bača, S. Jendroľ, M. Miller and J. Ryan, On irregular total labellings, Discrete Mathematics, 307 1378-1388 (2007).

[2] M. Bača and M.K. Siddiqui, Total edge irregularity strength of generalized prism, Applied Mathematics and Computation, 235 168-173 (2014).

[3] G.S. Bloom and S.W. Golomb, Numbered complete graphs, unusual rulers and assorted applications, in: Theory and applications of Graphs in: Lecture Notes in Math, 642 53-65 (1978), Springer-Verlag, New York.

[4] R. Cattel, Graceful labellings of paths, Discrete Mathematics, 307-24 3161-3176 (2007).

[5] S.N. Daoud, Edge odd graceful labeling of some path and cycle related graphs, $A K C E$ International Journal of Graphs and Combinatorics, 14 178-203 (2017).

[6] J.A. Gallian, A dynamic survey of graph labelling, The Electronic Journal of Combinatorics, 17 (2017).

[7] J. Ivančo and S. Jendrol̆, Total edge irregularity strength of trees, Discussiones Mathematicae Graph Theory, 26 449-456 (2006).

[8] S. Jendroľ, J. Misšuf and R. Soták, Total edge irregularity strength of complete graphs and complete bipartite graphs, Elec. Notes Discrete Math., 28 281-285 (2007).

[9] P. Jeyanthi and S. Philo, Odd harmonious labeling of some new families of graphs, Electronic Notes in Discrete Mathematics, 48 165-168 (2015).

[10] P. Jeyanthi and K.J. Daisy, $Z_{k}$-Magic labeling of open star of graphs, Bulletin of The International Mathematical Virtual Institute, 7 243-255 (2017).

[11] S.P. Lo, On edge graceful labelings of graphs, Congr. Numer. 50 231-241 (1985).

[12] R.W. Putra and Y. Susanti, On total edge irregularity strength of centralized uniform theta graphs, AKCE International Journal of Graphs and Combinatorics, 15(1) 7-13 (2018).

[13] R.W. Putra, R.W. and Y. Susanti, The total edge irregularity strength of uniform theta graphs, Journal of Physics: Conference Series, 1097 (2018) 012069.

[14] L. Ratnasari and Y. Susanti, Total edge irregularity strength of ladder related graphs, Asian-European Journal of Mathematics, 13(4), (2020) 2050072.

[15] L. Ratnasari, S. Wahyuni, Y. Susanti, D. Junia Eksi Palupi and B. Surodjo, Total edge irregularity strength of arithmetic book graphs, Journal of Physics: Conference Series 1306(1), (2019) 012032. 
[16] A. Solairaju and K. Chithra, Edge-odd graceful graphs, Electronic Notes in Discrete Mathematics, 33 15-20 (2009).

[17] Y. Susanti, I. Ernanto, B. Surodjo, On Some New Edge Odd Graceful Graphs, AIP Conference Proceedings, 2019, 2192, 040016.

[18] W.D. Wallis, Magic graphs, (2001) Springer, New York. 